Baltic Astronomy, vol.23, 71-91, 2014

\title{
DYNAMICS OF COSMIC NEUTRINOS IN GALAXIES
}

\author{
A. Sapar \\ Tartu Observatory,61602 Tõravere,Estonia; sapar@ao.ee
}

Received: 2014 June 11; accepted: 2014 July 2

\begin{abstract}
The cosmic background of massive (about $1 \mathrm{eV}$ rest-energy) neutrinos can be cooled to extremely low temperatures, reaching almost completely degenerated state. The Fermi velocity of the neutrinos becomes less than 100 $\mathrm{km} / \mathrm{s}$. The equations of dynamics for the cosmic background neutrinos are derived for the spherical and axisymmetrical thin circular disk galaxies. The equations comprise the gravitational potential and gravity of the uniform baryonic disk galaxies. Then the equations are integrated analytically over the disk radius. The constant radial neutrino flux in spherical galaxies favors formation of the wide unipotential wells in them. The neutrino flux in the axisymmetrical galaxies suggests to favor the evolution in the direction of a spherically symmetrical potential. The generated unipotential wells are observed as plateaux in the velocity curves of circular stellar orbits. The constant neutrino density at galactic centers gives the linear part of the curves. The derived system of quasilinear differential equations for neutrinos in the axisymmetrical galaxies have been reduced to the system of the Lagrange-Charpit equations: the coupled differential equations, specifying the local neutrino velocities and dynamics of motion along trajectories, and an additional interconnected equation of the neutrino mass conservation, which can be applied for the determination of density of the neutrino component in galaxies.
\end{abstract}

Key words: cosmology: galaxies, theory, dark matter, neutrinos

\section{INTRODUCTION}

Different conjectures have been proposed on the nature of the dark mass in galaxies. Here we will refer only to some recent generalizing suggestions.

The most simple assumption is that galaxies have very extended baryonic halos. In these low-density halos due to trend of virialization different particles can have different temperatures and high ionization degrees. As it has been demonstrated, based on the X-ray observations aboard the Chandra X-ray Space Observatory, the circumgalactic medium of the Milky Way extends to over $100 \mathrm{kpc}$ and its baryonic mass is over $10^{10}$ solar masses (Gupta et al. 2012 and references therein). The estimates show that the extended circumgalactic halos can give some additional contribution to the baryonic matter. This mass can be of the local origin or can be expelled from our Galaxy. The same situation can take place in other galaxies, but for them similar X-ray studies are needed.

Another possibility, which till now is not fully excluded, is based on the neutrinos with the rest-mass. This possibility has been analyzed in many papers. It is 
not excluded that the rest energy of neutrinos is of the order $1 \mathrm{eV}$, this value is accepted in the present study. Even if the mean rest mass of neutrinos is considerably smaller, the equations derived in the present paper do not lose their significance and can be applied for other weak-interacting particles. An additional possibility is the existence of the hypothetic sterile neutrinos and their role in generating of dark matter (Canetti et al. 2013). These authors generalized the standard Langrangian model of leptons to the Lagrangian, which takes into account also the interaction with the sterile neutrinos and with the Higgs boson doublet. In this way they succeeded to explain a generation of the dark matter and baryon asymmetry of the universe and concluded the presence of similar asymmetry for leptons. Also, other different exotic particles (say, WIMPs, axions) have been proposed as the hitherto mysterious dark matter in galaxies and their clusters.

The problem about the role of neutrino contribution to the dark matter has been on agenda for decades. The main difficulty in the study of their role is that the rest-mass of neutrinos is so far unknown regardless of many efforts to determinate its value. One of the most effective limiters of possible rest-mass values are the cosmological studies and studies of neutrino background as the dark mass contribution in galaxies and their clusters. Presently we confine our analysis on the possibility that the main particles in the dark mass are massive neutrinos within their standard weak interaction model.

The cosmological redshift factor $1+z$ of all background particles is coupled to their momenta. Thus, at non-relativistic velocities the massive neutrinos should have cooled down as squared relative to the momentum $p \propto(1+z)$ in the energy expression. Therefore the present cosmic neutrino background must have very low temperature and this leads to almost complete degeneration. The neutrinos with the $1 \mathrm{eV}$ rest energy have velocities, which are now less than $100 \mathrm{~km} / \mathrm{s}$. Such cosmic neutrino background is favorable for its role and interplay in the formation and evolution of galaxies.

A peculiarity of the neutrinos is that their orbits or trajectories throughout the galaxies can be treated as individual ones across the averaged gravity field. Thus, the hydrodynamical mass conservation equations of neutrinos are coupled with their trajectories. This circumstance couples computing of the neutrino constituent density in galaxies with their trajectories.

\section{DEGENERATED COSMIC NEUTRINO BACKGROUND}

The present temperature of the cosmic microwave background is $T_{\gamma}^{0}=2.725$ $\mathrm{K}$ and the thermal number density of photons $N_{\gamma}^{0}=410.5$. The corresponding temperature of the massless neutrino background should be $T_{\nu}^{0}=(4 / 11)^{1 / 3} T_{\gamma}^{0}=$ $0.71376 T_{\gamma}^{0}=1.945 \mathrm{~K}$ and the corresponding number density of neutrinos is $N_{\nu}^{s}=$ $7 N_{\gamma}^{s} / 8$ for each of their species $s$. Thus, the thermal number density of a neutrino species relative to the number density of the photon spin state is

$$
N_{\nu}^{0}=\frac{7}{8}\left(\frac{T_{\nu}^{0}}{T_{\gamma}^{0}}\right)^{3} \frac{N_{\gamma}^{0}}{2}=65.3 \mathrm{~cm}^{-3} .
$$

Since till now $p_{\nu}=E_{\gamma} / c$ is valid (Sapar 2011), for the kinetic energy of low-velocity 
neutrino background we can simply write:

$$
E_{\nu}=k T_{\nu}=\frac{p_{\nu}^{2}}{2 m_{\nu}}=\frac{E_{\gamma}^{2}}{2 m_{\nu} c^{2}}=5.517 \cdot 10^{-8} \mathrm{eV} .
$$

Taking into account that $1 \mathrm{eV} / k=11604.5 \mathrm{~K}$, we obtain

$$
T_{\nu}=3.199 \cdot 10^{-4} \mathrm{~K} .
$$

Thus, the corrected temperature of the present neutrino background is

$$
T_{\nu}^{c}=\left(\frac{4}{11}\right)^{1 / 3} T_{\nu}=2.284 \cdot 10^{-4} \mathrm{~K} .
$$

The Fermi energy of completely degenerated (all the lower Pauli states filled at the absolute zero temperature) neutrinos is

$$
E_{F}=\frac{\pi^{2} \hbar^{2}}{2 m_{\nu}}\left(\frac{3 N}{\pi}\right)^{2 / 3},
$$

where $N$ is the number density of each neutrino species. Thus, if $m_{\nu} c^{2}=1 \mathrm{eV}$ and for the species of neutrino background we may use equation (1), then computing formally the classical equivalent temperature of the Fermi energy at the present epoch, we obtain:

$$
\frac{E_{F}}{k}=T_{F}=3.207 \cdot 10^{-4} \mathrm{~K} .
$$

The numerical closeness of the $T_{F}$ and $T_{\nu}^{c}$ values, which are obtained in quite different ways, is amazing. This means that the neutrino background is, and for a long time has been, almost completely degenerated or, in other words, extremely cold. The corresponding present Fermi velocity of the background $1 \mathrm{eV}$ rest-mass neutrinos is

$$
v_{0}=\left(\frac{2 E_{F}}{m_{\nu}^{1}}\right)^{1 / 2}=44.4 \mathrm{~km} / \mathrm{s} .
$$

Let us now estimate the critical contribution of neutrinos to the cosmological density of matter. If the rest energy of neutrinos is $1 \mathrm{eV}$, then their mass is $m_{\nu}^{1}=1.7838 \cdot 10^{-33} \mathrm{~g}$. The general contribution of neutrinos by their six species is given by

$$
\rho_{\nu}^{0}=6 m_{\nu}^{1} N_{\nu}^{0}=6.985 \cdot 10^{-31} \mathrm{~g} / \mathrm{cm}^{3} .
$$

This is about $7 \%$ of the general matter density, corresponding to the present value of the Hubble constant, $H_{0}=73 \mathrm{~km} /(\mathrm{s} \mathrm{Mpc})$. From the neutrino oscillations it has been concluded, that the mass differences of $\nu_{e}, \nu_{\mu}$ and $\mu_{\tau}$ are small, what justifies to take the same masses for all neutrino species (flavours).

\section{RADIALLY MOVING NEUTRINO BACKGROUND IN SPHERICAL GALAXIES}

Let us look now at the problem of dark mass in galaxies in the aspect of neutrino contribution. First, for simplicity we start here with the description of 
physics of a spherical galaxy with neutrino constituent. In this case it is useful to start from the mass conservation law and from the stationarity condition (lack of time dependance) of the neutrino flux, $F$, which must be a constant on every spherical surface, i.e.

$$
4 \pi r^{2} \rho_{\nu} v_{\nu}=F .
$$

The boundary velocities of neutrinos are considered to be $v_{a}=v_{0}$.

Denoting the radial neutrino mass density as

$$
\sigma_{\nu}=4 \pi r^{2} \rho_{\nu}
$$

and the radial baryonic mass density as

$$
\sigma_{b}=4 \pi r^{2} \rho_{b},
$$

the following radial neutrino flux is obtained:

$$
\sigma_{\nu} v_{\nu}=\sigma_{\nu} \dot{r}=F .
$$

This is the integrated equation of mass conservation along the radial neutrino trajectories.

Using the radial mass densities we obtain

$$
M_{\nu}(r)=\int_{0}^{r} \sigma_{\nu} d r, \quad M_{b}(r)=\int_{0}^{r} \sigma_{b} d r .
$$

The dynamics of neutrino velocities along the trajectories is determined by the gravity, which in the present case has the form:

$$
\ddot{r}=g_{r}=-\frac{G\left(M_{\nu}(r)+M_{b}(r)\right)}{r^{2}} .
$$

In these equations $r$ is the current radius and $M$ is the total mass in the sphere for neutrinos, $\nu$, and baryons, $b$. This leads to the conclusion that

$$
\frac{d\left(r^{2} \ddot{r}\right)}{d r}=-G 4 \pi r^{2}\left(\rho_{\nu}+\rho_{b}\right)=-G\left(\sigma_{\nu}+\sigma_{b}\right) .
$$

Let us consider now some main features of the neutrino background. At the galactic center, $d \rho / d r=0$ must hold. This means that there are neutrino cores with the constant density $\rho_{\nu}^{0}$. Thus, in this region

$$
M_{\nu}(r)=\frac{4 \pi r^{3}}{3} \rho_{\nu}^{0}
$$

and the expression for neutrino velocities is

$$
v_{\nu}=\frac{F}{4 \pi r^{2} \rho_{\nu}^{0}} .
$$

The corresponding gravitational potential relative to the galactic center is

$$
U_{\nu}(r)=\frac{M_{\nu}(r)}{r}=\frac{4 \pi r^{2}}{3} \rho_{\nu}^{0} .
$$


Consequently, for the circular stellar orbits we have

$$
\frac{v_{b}^{2}}{2}=U_{\nu}(r)=\frac{M_{\nu}(r)}{r}=\frac{4 \pi r^{2}}{3} \rho_{\nu}^{0} .
$$

Thus, in the galactic nuclear region the orbital velocity due to neutrino contribution is proportional to the radial distance $r$. Also, the central regions of galaxies can be treated taking into account the neutrino contribution to dark mass.

There is also a region where $\sigma_{\nu}=\sigma_{\nu}^{c}$ is constant. In this region the central potential of neutrino contribution is constant, namely

$$
U_{\nu}^{c}=\sigma_{\nu}^{c} .
$$

This region corresponds to the observed plateaux in circular stellar orbits.

The value of the neutrino flux $F$ can be estimated from the accretion sphere, generated by neutrinos

$$
F=4 \pi r_{a}^{2} \rho_{a} v_{a} .
$$

The mass has been accreted from the volume having the radius $r_{a}$ and the present cosmic neutrino background density $\rho_{a}$. Thus, we can write

$$
M_{\nu, a}=\frac{4 \pi r_{a}^{3}}{3} \rho_{a} .
$$

The largest radius of the accretion (crossover) sphere, $r_{a}$, for degenerate neutrinos with maximal velocity $v_{a}=v_{0}$ can be estimated from

$$
H_{0}=\frac{\dot{R}_{0}}{R_{0}}=\frac{v_{a}}{r_{a}} .
$$

We obtain that presently the accretion radius $r_{a}$ for neutrinos with the rest energy $1 \mathrm{eV}$ is about $r_{a}=0.62 \mathrm{Mpc}$. The larger is the neutrino mass, the smaller are the Fermi velocity and the radius of the accretion sphere.

Here we have ignored the time-dependence terms and the non-spherical distribution of the baryonic and of the neutrino matter. In addition, simplification has been made using instead of the velocity distribution of neutrinos their velocity corresponding to the Fermi energy. We hope that these simplifications do not have drastic influence on the results obtained. We also hope that the Karlsruhe experiment KATRIN of the tritium $\beta$-decay, i.e. the generation of electron antineutrinos in the process ${ }^{3} \mathrm{H} \Rightarrow{ }^{3} \mathrm{He}+e+\bar{\nu}_{e}$, will solve the long-standing problem of the value of $\nu_{e}$ mass in the near future.

\section{NON-RADIAL NEUTRINO TRAJECTORIES AND THEIR TRANSFER IN SPHERICAL GALAXIES}

Now we take into account that neutrinos have initial angular distribution and respectively the distribution of the angular momenta. Let the angular momentum of neutrinos per unit mass be $L$. Thus, for the azimuthal velocity component $v_{\psi}$ of neutrinos we can write

$$
v_{\psi}=r \dot{\psi}=\frac{L}{r} .
$$


This equation justifies the replacement of the time $t$ with an independent variable $\psi$ which is the azimuthal angle of neutrino velocity in the orbital plane. Its initial value is specified at the boundary of the accretion sphere. The trajectories we consider in the first quadrant of the azimuth.

Using the equation (24) we can eliminate the time derivatives along neutrino trajectories, substituting

$$
\frac{d}{d t}=\frac{L}{r^{2}} \frac{d}{d \psi}
$$

The general equation of the motion in the central-symmetrical gravity field has the form

$$
\ddot{r}-\frac{v_{\psi}^{2}}{r}=g_{r}=-\frac{G\left(M_{\nu}(r)+M_{b}(r)\right)}{r^{2}} .
$$

Based on these equations, we can write

$$
\frac{L^{2}}{r^{2}} \frac{d r}{d \psi}\left(\frac{1}{r^{2}} \frac{d r}{d \psi}\right)-\frac{L^{2}}{r^{3}}=g_{r}
$$

To simplify the equation, it is useful to introduce a new argument

$$
u=\frac{1}{r}
$$

Multiplying equation (26) by $d u / d \psi$ and integrating over $\psi$ we obtain for any trajectory of neutrinos (like for stellar orbits in galaxies)

$$
\left(\frac{d u}{d \psi}\right)^{2}+u^{2}=\frac{A^{2}}{L^{2}}, \quad A^{2}=2\left(E_{a}+\Delta E\right) .
$$

In this equation the constant of integration $E_{a}$ is the boundary value of the kinetic energy per unit mass of neutrinos and $\Delta E$ is its change in the gravitation field, given by

$$
E_{a}=\frac{v_{a}^{2}}{2}, \quad \Delta E=\int_{r}^{r_{a}} g_{r} d r .
$$

The boundary value of the angular momentum per unit mass of neutrinos is specified by

$$
L=v_{a} r_{a} \sin \psi_{a},
$$

where $\psi_{a}$ is the angle between the directions to the center and the velocity vector at the accretion sphere boundary.

The closest point of the neutrino trajectory to the center of stellar system (pericenter) is specified by $\frac{d u}{d \psi}=0$ giving the well-known equation

$$
u_{L}=\frac{1}{r_{L}}=\frac{A}{L}=\frac{A}{r_{a} v_{a} \sin \psi_{a}} .
$$

Equation (29) is the well-known geometrical equation for the particle trajectories. 
In order to obtain more general treatment of trajectories we must use the original equation of a trajectory, i.e. equation (26). In addition, we must take into account that the components of the velocity are given by

$$
v_{r}=\dot{r}, \quad v_{\psi}=r \dot{\psi} .
$$

For the radial acceleration of the neutrino trajectory we have

$$
\ddot{r}=-\frac{G\left(M_{r}+m_{r}\right)}{r^{2}}+\frac{L^{2}}{r^{3}}
$$

and for the azimuthal angle in the case of radial gravity field, cf. equation (93), it follows that the angular acceleration is specified by

$$
r \ddot{\psi}+2 \dot{r} \dot{\psi}=0,
$$

from where

$$
\ddot{\psi}=-\frac{2 \dot{r} \dot{\psi}}{r} .
$$

The characteristic curves of these quasilinear differential equations, which correspond to the neutrino trajectories, are the Lagrange-Charpit equations

$$
\frac{d r}{\dot{r}}=\frac{d \psi}{\dot{\psi}}=\frac{d \dot{r}}{\ddot{r}}=\frac{d \dot{\psi}}{\ddot{\psi}}=d \tau .
$$

Here the expression for $\ddot{r}$ must be taken from (34), for $\ddot{\psi}$ from (36), and $\tau$ is the integration parameter which for the given problem is normal to be chosen as coinciding with time interval in the neutrinos transfer.

Along the neutrino trajectories the following equation holds:

$$
\frac{d}{d t}=\dot{r} \frac{\partial}{\partial r}+\dot{\psi} \frac{\partial}{\partial \psi} .
$$

In addition to the equations of trajectories, it is necessary to use the mass conservation law for neutrinos. In the spherical coordinates the divergence of neutrino flux has the form

$$
\frac{1}{r^{2}} \frac{\partial\left(\rho r^{2} v_{r}\right)}{\partial r}+\frac{1}{r \sin \psi} \frac{\partial\left(\rho \sin \psi v_{\psi}\right)}{\partial \psi}=0
$$

The circumstance that the characteristic curves for the partial differential equations (34) and (35) are the neutrino trajectories becomes apparent taking into account that for trajectories must hold

$$
\frac{d r}{d \psi}=\frac{\dot{r}}{\dot{\psi}} .
$$

This shows that the Lagrange-Charpit equations specify the neutrino trajectories.

Equation (39) can be written in the form

$$
\dot{r} \frac{\partial \rho}{\partial r}+\dot{\psi} \frac{\partial \rho}{\partial \psi}=\dot{\rho},
$$


where

$$
\dot{\rho}=-\rho\left(\frac{\partial\left(r^{2} \dot{r}\right)}{r^{2} \partial r}+\frac{1}{\sin \psi} \frac{\partial(\sin \psi \dot{\psi})}{\partial \psi}\right) .
$$

From this equation we find for the characteristic curve of the neutrino density an additional usual differential equation, in addition to other Lagrange-Charpit equations (37), namely:

$$
\frac{d \rho}{\dot{\rho}}=d \tau .
$$

The run of the $\rho$ value along the neutrino trajectory is given by this expression where time derivatives in $\dot{\rho}$ are found from the equations of the neutrino trajectory. From the expression (42) it follows that far from the galactic center the gradient of the radial neutrino flux is slowly changing along the neutrino trajectories, i.e. there are wide approximately unipotential wells.

If $v_{\psi}=0$, then for the radial neutrino flux we obtain the familiar result

$$
4 \pi \rho r^{2} v_{r}=F,
$$

where the constant $F$ is the radial neutrino flux throughout a spherical galaxy.

To illustrate approximately the general mass contribution by neutrinos in the case of non-zero angular momentum, we accept tentatively the neutrino mass distribution in the sphere with radius $r$ by

$$
m_{r}=m_{0} \frac{r^{3}}{\left(r_{o}+r\right)\left(r+r_{i}\right)^{2}},
$$

where $r_{o}$ is approximately the value of the outer boundary distance of the flat galactic gravitational potential well and $r_{i}$ is the inner boundary distance of the transition to its constant density core. This can be treated as slightly modified version of the Navarro-Frenk-White density profile, which has been established by the N-body simulations of galaxy formation.

\section{AXISYMMETRICAL FLAT CIRCULAR DISK GALAXIES}

Now let us turn to the gravitational potential of the uniform gravitating circular plane disk in a rotating galaxy. In this case the gravitational potential in the mesh points is given by the integral

$$
U(\mathbf{D})=\frac{G M}{\pi R} \int_{0}^{1} \int_{0}^{2 \pi} \frac{d \varphi r d r}{|\mathbf{D}-\mathbf{r}|}
$$

In this equation, $M$ is the total mass of the disk. The distance vectors are specified by a coordinate frame choice. Without any loss of generality, we can take for the current mesh point $\mathbf{D}=(x, 0, z)$ and for the disk points $\mathbf{r}=(r \cos \varphi, r \sin \varphi, 0)$. In addition, we are scaling the distances to dimensionless quantities, expressed in the units of disk radius $R$. This integral in the chosen coordinates simplifies to

$$
U(\mathbf{D})=\frac{2 G M}{\pi R} \int_{0}^{1} \int_{0}^{\pi} \frac{d \varphi r d r}{\left(D^{2}+r^{2}-2 r x \cos \varphi\right)^{1 / 2}}, \quad x=D \sin \psi
$$


This equation can be written in the form

$$
U(\mathbf{D})=\frac{2 G M}{\pi R} \int_{0}^{\pi} d \varphi \int_{0}^{1} \frac{r d r}{Z(r)} .
$$

Here we introduced the notation

$$
Z^{2}(r)=A r^{2}+B r+C
$$

where

$$
A=1, \quad B=-2 x \cos \varphi, \quad C=D^{2} .
$$

The integration discriminant of $Z(r)$ is

$$
\Delta=4 A C-B^{2}=4\left(x^{2}+z^{2}\right)-4 x^{2} \cos ^{2} \varphi=4\left(z^{2}+x^{2} \sin ^{2} \varphi\right) .
$$

Whereas generally $\Delta>0$, excluded in the disk plane, $z=0$, at $\varphi=0$ then analytical integration gives

$$
J(r)=\int \frac{r d r}{Z(r)}=Z(r)-\frac{B}{2} \ln Q(r),
$$

where

$$
Q(r)=2 Z(r)+2 r+B .
$$

Using the needed integration limits we obtain

$$
Z(0)=D, \quad Z(1)=\left(1+B+D^{2}\right)^{1 / 2} .
$$

From here, substituting into (52) the limits of integration, we obtain

$$
J(1)=Z(1)-\frac{B}{2} \ln Q(1), \quad J(0)=Z(0)-\frac{B}{2} \ln Q(0) .
$$

In these equations

$$
Q(1)=2 Z(1)+2+B, \quad Q(0)=2 D+B .
$$

Totally we have for the integral, which depends on the azimuthal angle $\varphi$, the expression

$$
Y(\varphi)=J(1)-J(0)=Z(1)-Z(0)+\frac{B}{2} \ln \left(\frac{Q(0)}{Q(1)}\right)
$$

Next we will study the integrals over $\varphi$. The most important is the integral

$$
\Gamma=\int_{0}^{\pi}(Z(1)-Z(0)) d \varphi .
$$


To make evident its physical properties, we will multiply it by $(Z(1)+Z(0))$, obtaining

$$
\Gamma=\int_{0}^{\pi} \frac{1+B}{Z(1)+D} d \varphi
$$

In more detailed form we can write

$$
\Gamma=\int_{0}^{\pi} \frac{1-2 D \sin \psi \cos \varphi}{\left(1-2 D \sin \psi \cos \varphi+D^{2}\right)^{1 / 2}+D} d \varphi
$$

In its turn this integral can be written in the form

$\Gamma=\int_{0}^{\pi / 2} \frac{1-2 D \sin \psi \cos \varphi}{\left(1-2 D \sin \psi \cos \varphi+D^{2}\right)^{1 / 2}+D} d \varphi+\int_{0}^{\pi / 2} \frac{1+2 D \sin \psi \cos \varphi}{\left(1+2 D \sin \psi \cos \varphi+D^{2}\right)^{1 / 2}+D} d \varphi$.

From here it follows that in the galactic center $\Gamma=\pi$ and at large distances $D$ it tends to $\pi / 2 D$ as needed.

Now we will study the integral

$$
W=\int_{0}^{\pi} \frac{B}{2} \ln \left(\frac{Q(0)}{Q(1)}\right) d \varphi=-x \int_{0}^{\pi} \cos \varphi \ln \left(\frac{Q(0)}{Q(1)}\right) d \varphi
$$

To get rid of singularity, in the expression $Q(0)$ at integrating over the $\varphi$ in the disk plane, we multiply for the first quarter of $\varphi$ the nominator and denominator by $2 Z(1)-2-B$, obtaining for both quarters the expressions free of singularity, namely

$$
W=-x \int_{0}^{\pi / 2} \cos \varphi \ln \left(\frac{2 Z(1)-2-B}{2 D-B}\right) d \varphi-x \int_{\pi / 2}^{\pi} \cos \varphi \ln \left(\frac{2 D+B}{2 Z(1)+2+B}\right) d \varphi .
$$

Finally, for the gravitational potential of the uniform circular disk we obtain a quite simple expression

$$
U(\mathbf{D})=\frac{2 G M}{\pi R}(\Gamma+W) .
$$

Some comments on $Y(\varphi)$. At large distances we can take $Z(1)=D$, obtaining

$$
Y(\varphi)=\frac{1}{D}
$$

At the points very close to the galactic center, $D \Rightarrow 0$ and $Y(\varphi)=1$. Consequently, $U(0)=2 G M / R$.

The next task is to compose the equation for the galaxies having arbitrary axisymmetrical density profiles. We start with adding another disk at the distance $h$ in the direction of $z$. The numerical values of its distances we express in units of 
the central disk radius $R$. In order to use the integrals found, we must recalibrate to these units the coordinates for the potential at each current space point. We mark the new coordinates by the corresponding primed notations. Using the scaling factor $S$, in the scaled coordinates we obtain the equations

$$
S=\frac{R}{R^{\prime}}, \quad x^{\prime}=S x, \quad z^{\prime}=S(z-h), \quad D^{\prime 2}=x^{\prime 2}+z^{\prime 2}, \quad \cos \psi^{\prime}=\frac{z^{\prime}}{D^{\prime}} .
$$

The corresponding gravitational potential of the other coaxial disk can now be expressed in the form

$$
U^{\prime}(h)=\frac{2 G M^{\prime}}{\pi R^{\prime}} \int_{0}^{1} \int_{0}^{\pi} \frac{d \varphi r d r}{\left(D^{\prime 2}+r^{2}-2 r x^{\prime} \cos \varphi\right)^{1 / 2}} .
$$

In this way we obtain for the total gravitational potential of galaxy, approximated by circular coaxial disks of homogeneous density the following equation

$$
U\left(h^{*}\right)=\int_{-h^{*}}^{h^{*}} U^{\prime}(h) d h .
$$

As a general comment, the computations starting from densities instead of gravitational potentials are prefered, because then getting of negative densities is automatically excluded.

Let us now compare shortly our equations of gravitational potential of the homogeneous disk with the equations derived in other studies. The circumstance that integration along the radial coordinate can be acomplished analytically has been found by Lass \& Blitzer (1983). For the gravitational potential they derived more complicated expressions via complete elliptic integrals of the 1st, 2nd and 3rd kind, the last having two arguments. The same functions and some more different special functions have been used for finding the gravitational potential in more general axisymmetrical cases of the mass distribution by Conway (2000). Some other special cases have been analyzed by Huré \& Hersant (2011), which are primarily suitable for study of the structure of the accretion disks. The compact equations for the gravitational potential derived in the present paper are free of singularities.

Some authors used a possibility to express the gravitational potential as a power series, expressed via the Legendre polynomials. This method is extremely slowly converging and the equations are too clumsy.

\section{GRAVITY IN FLAT CIRCULAR DISK GALAXIES}

As we have demonstrated, the singularity in the expression of the potential $U(\mathbf{D})$ can be removed applying a simple arithmetical transformation. However, for the gravity singularities appear everywhere on surfaces of the disk galaxies. Starting from (48) we can write the radial component of gravity in the form

$$
g_{D}=\frac{\partial U(\mathbf{D})}{\partial D}=-\frac{2 G M}{\pi R}\left(D I_{1}-\sin \psi I_{2}\right),
$$


where the integrals

$$
I_{n}=\int_{0}^{\pi} q_{n} \cos ^{n-1} \varphi d \varphi, \quad q_{n}=\int_{0}^{1} \frac{r^{n} d r}{Z(r)^{3}} .
$$

In a similar way, for the angular component of gravity we obtain

$$
g_{\psi}=\frac{\partial U(\mathbf{D})}{D \partial \psi}=\frac{2 G M}{\pi R} \cos \psi I_{2} .
$$

For $I_{1}$ the analytical expression of indefinite integral $q_{1}(r)$ is

$$
q_{1}(r)=-2 \frac{B r+2 C}{Z(r) \Delta}
$$

and for $I_{2}$ the expression of $q_{2}(r)$ is

$$
q_{2}(r)=\frac{\left(2 B^{2}-4 A C\right) r+2 B C}{A Z \Delta}+\frac{\ln (Q(r))}{A^{3 / 2}} .
$$

In the present case $A=1$. For the needed definite integrals $q_{n}$ we obtain

$$
q_{n}=q_{n}(1)-q_{n}(0) .
$$

If $\psi=0$, then $B=0$ and

$$
q_{1}(r)=-\frac{4 C}{4 A C\left(r^{2}+C\right)^{1 / 2}} .
$$

For $\psi=0$ it also follows that

$$
\frac{\partial U(\mathbf{D})}{\partial D}=\frac{2 G M}{R}\left(1-\frac{D}{\sqrt{1+D^{2}}}\right)=\frac{2 G M}{R} \frac{1}{\sqrt{1+D^{2}}\left(D+\sqrt{1+D^{2}}\right)} .
$$

This is known expression showing that at small values of $D$ the radial component of gravity along the ordinate axis is almost constant and at large distances it tends to $G M /\left(R D^{2}\right)$ and at the galactic center it equals to $2 G M / R$.

Along the abscissa axis, where $\psi=\pi / 2$, the situation is much more complicated.

The gravity can be written by

$$
\mathbf{g}=\left(\frac{\partial U}{\partial D}, \frac{\partial U}{D \partial \psi}\right)=\frac{\partial U}{\partial D} i_{D}+\frac{\partial U}{D \partial \psi} i_{\psi}=g_{D} i_{D}+g_{\psi} i_{\psi}
$$

Thus,

$$
g^{2}=g_{D}^{2}+g_{\psi}^{2} .
$$

It is reasonable try to present the obtained equations in the form where their physical behavior is seen more explicitely. At large distances it is reasonable to recalibrate quantities $A, B, C$ by

$$
A^{\prime}=A / D^{2}, \quad B^{\prime}=B / D^{2}, \quad C^{\prime}=C / D^{2} .
$$


The corresponding discriminant is $\Delta^{\prime}=\Delta / D^{4}$ and $Z^{\prime}=Z / D$. In such calibration $C^{\prime}=1$, and we obtain

$$
q_{1}=\frac{2}{D^{3}} \frac{2 Z_{1}^{\prime}-B^{\prime}-2}{Z_{1}^{\prime} \Delta^{\prime}}=\frac{4}{D^{3}} \frac{1}{Z_{1}^{\prime}\left(2 Z_{1}^{\prime}+B^{\prime}+2\right.} .
$$

At large distances $Z^{\prime} \Rightarrow 1, B^{\prime} \Rightarrow 0$ and thus $q_{1} \Rightarrow D^{-3}$. The correction term $q_{2}$ at large distances also behaves as $q_{2} \propto D^{-3}$.

It is instructive to demonstrate in figures which are the correction terms for potential, for gravity and its components relative to the point mass gravitational field. Therefore we scale the functions by $G M / R=1$ and thereafter take into use the correction coefficients $C_{U}=U D, C_{g}=g D^{2}, C_{D}=g_{D} D^{2}$ and $C_{\psi}=g_{\psi} D^{2}$.

At large distances $D$ the quantities $C_{U}, C_{g}$ and $C_{D}$ tend to unity, but $C_{\psi}$ tends to zero. At the galactic center $C_{U}=0, C_{D}=0, C_{\psi}=0.5$ and $C_{g}=0.5$. The main features of their behavior are visualized in Figures $1-4$.

\section{NEUTRINO BACKGROUND IN FLAT CIRCULAR GALAXIES}

Let us start with some half-intuitional conjectures about distribution of the neutrino background in the flat galaxies, including the spiral galaxies. The first circumstance is that the increase of the neutrino energy corresponds to the local gravitational potential, but conservation of the momentum changes the direction of the neutrino flux. In addition, the flux contributions having different initial velocities overlap, giving their contribution to the local neutrino density. An essential difference relative to the spherically-symmetrical case is that there is a dependence of quantities on the polar angle $\psi$ from the galactic axis. In addition, the gravitational potential $\mathrm{U}$ must be replaced by the effective potential taking into account conservation of the $z$-component of the angular momentum. This means that we must also take into account the azimuthal, $\varphi$, component of angular momentum via corresponding contribution to the kinetic energy.

The Hamiltonian per unit mass of neutrinos is

$$
H=\frac{1}{2}\left(\dot{x}^{2}+x^{2} \dot{\varphi}^{2}+v_{z}^{2}\right)+U(x, z)=E .
$$

Here we have used the same coordinate frame choice as in the previous section, thus

$$
L_{z}=x^{2} \dot{\varphi},
$$

where $\varphi$ is the azimuthal angle. The corresponding contribution to the velocity of kinetic energy is

$$
V_{z}=\frac{L_{z}}{x}
$$

This means that to the potential energy in the distance change we should add the kinetic energy term, which grants conservation of the $z$-component of the angular momentum

$$
U_{L}=\frac{V_{z}^{2}}{2}=\frac{L_{z}^{2}}{2 x^{2}}
$$

To remove the azimuthal angle from further analysis, we introduce the effective potential $\Phi$ by

$$
\Phi=U(x, z)+U_{L}(x) .
$$




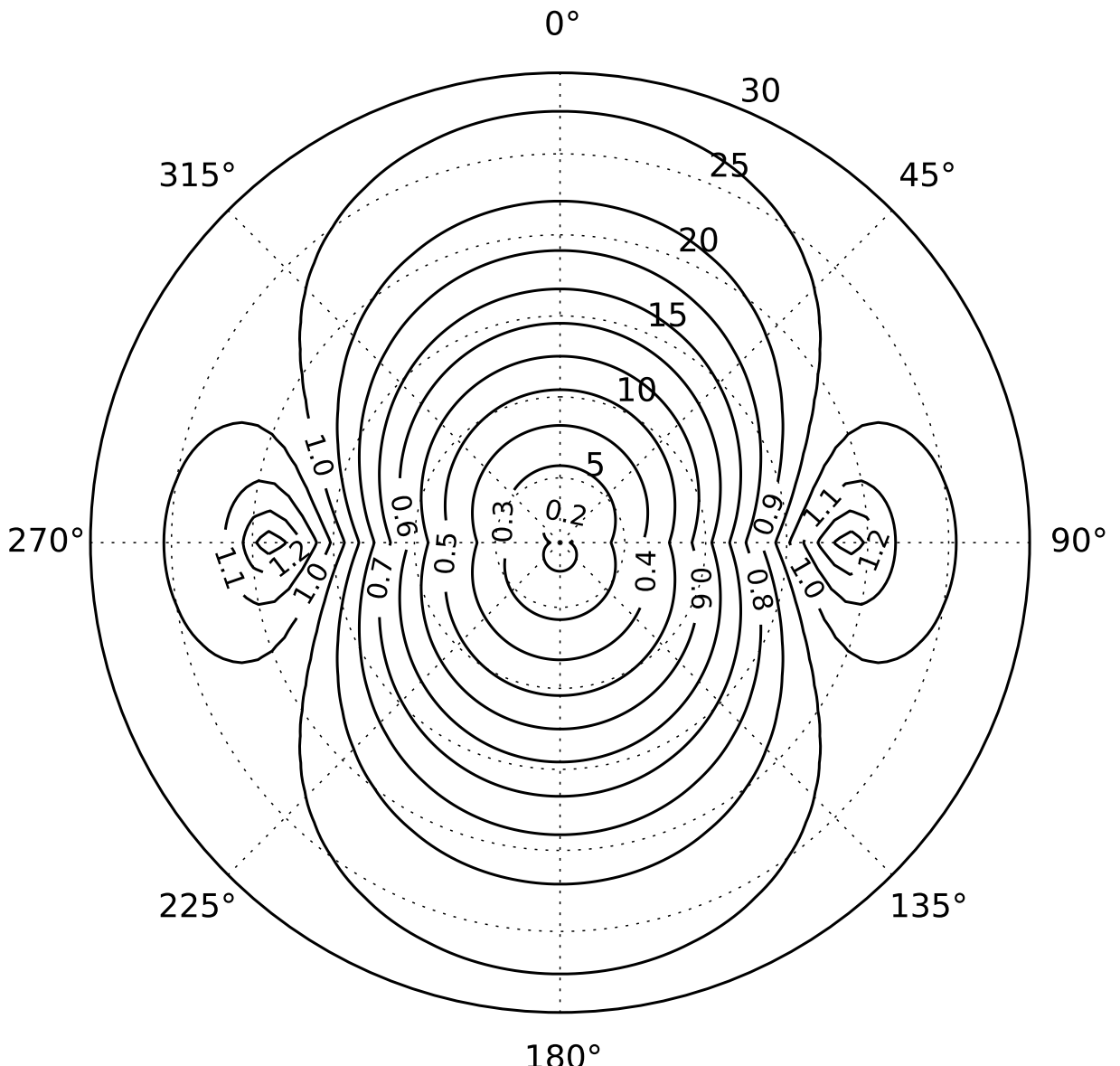

Fig. 1. Isolines of the correction factor of the gravitational potential, $C_{U}$. The coordinates are the scaled logarithm of the distance in the units of the circular disk galaxy radius, given in units $i D \cdot 0.05-1.0$. The distances from $D=0.1$ to $i D=30$, i.e. $D=\sqrt{10}$, have been covered. The maximum correction factors are on the galactic plane at $D=1$. At this point the asymmetry of mass distribution along the plane of circular disk galaxies has the maximal value. In the outer region free of isolines the value of $C_{U}$ has its asymptotic value approaching to 1.0. The central value of the correction factor is zero.

Starting from the accretion sphere we have

$$
L_{z}=r_{a} v_{a} \sin \psi_{a} .
$$

Now we find the trajectories of neutrinos. For the axisymmetrical galaxies effective gravity can be written in the form

$$
\mathbf{g}=-\nabla \Phi(x, z)
$$

i.e. as the gradient of the effective gravitational potential, depending on $x$ and $z$, which are the Cartesian coordinates. The distance vector $\mathbf{D}$ can be expressed 


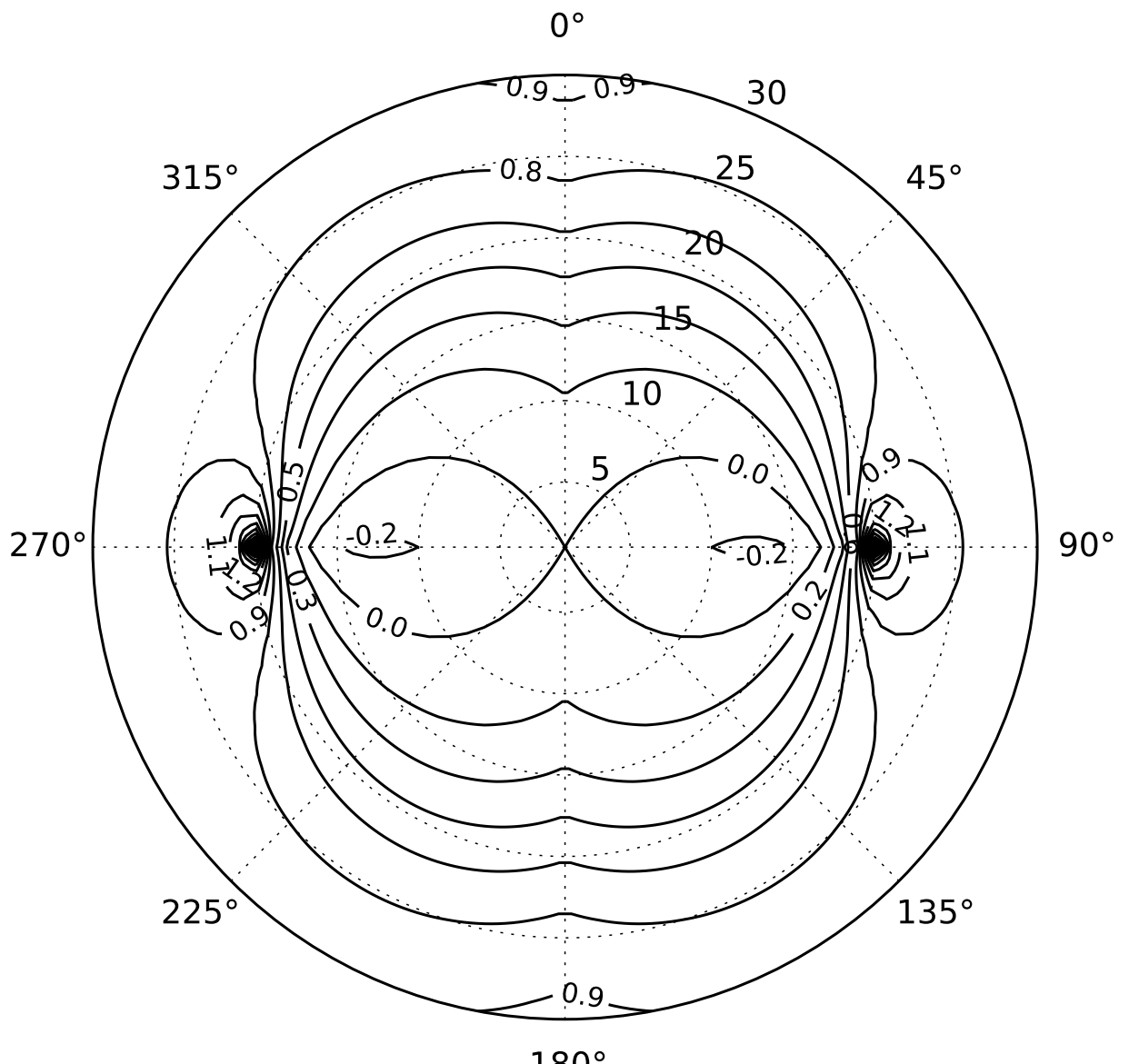

Fig. 2. Isolines of the correction factor of the radial gravity, $C_{D}$, being the radial gradient of gravitational potential. The coordinates are the same as in Figure 1. Similarly to the gravitational potential, in the outer region free of isolines the value of $C_{D}$ asymptotically approaches to 1.0, and the central value of the correction factor is zero.

also via the polar coordinates, which are more comfortable for the computation of neutrino trajectories, thus

$$
\mathbf{D}=x \mathbf{i}_{x}+z \mathbf{i}_{z}=D(\sin \psi, \cos \psi)=D \mathbf{i}_{D} .
$$

From here we find

$$
\dot{\mathbf{D}}=\dot{D} \mathbf{i}_{D}+D \dot{\psi}(\cos \psi,-\sin \psi)=\dot{D} \mathbf{i}_{D}+D \dot{\psi} \dot{\mathbf{i}}_{\psi} .
$$

Similarly

$$
\ddot{\mathbf{D}}=\left(\ddot{D}-D \dot{\psi}^{2}\right) \mathbf{i}_{D}+(D \ddot{\psi}+2 \dot{D} \dot{\psi}) \mathbf{i}_{\psi} .
$$

For the effective gravity we now obtain

$$
\mathbf{g}=\ddot{\mathbf{D}}=-\frac{\partial \Phi}{\partial x} \mathbf{i}_{x}-\frac{1}{D} \frac{\partial \Phi}{\partial z} \mathbf{i}_{z}=-\frac{\partial \Phi}{\partial D} \mathbf{i}_{D}-\frac{1}{D} \frac{\partial \Phi}{\partial \psi} \mathbf{i}_{\psi} .
$$




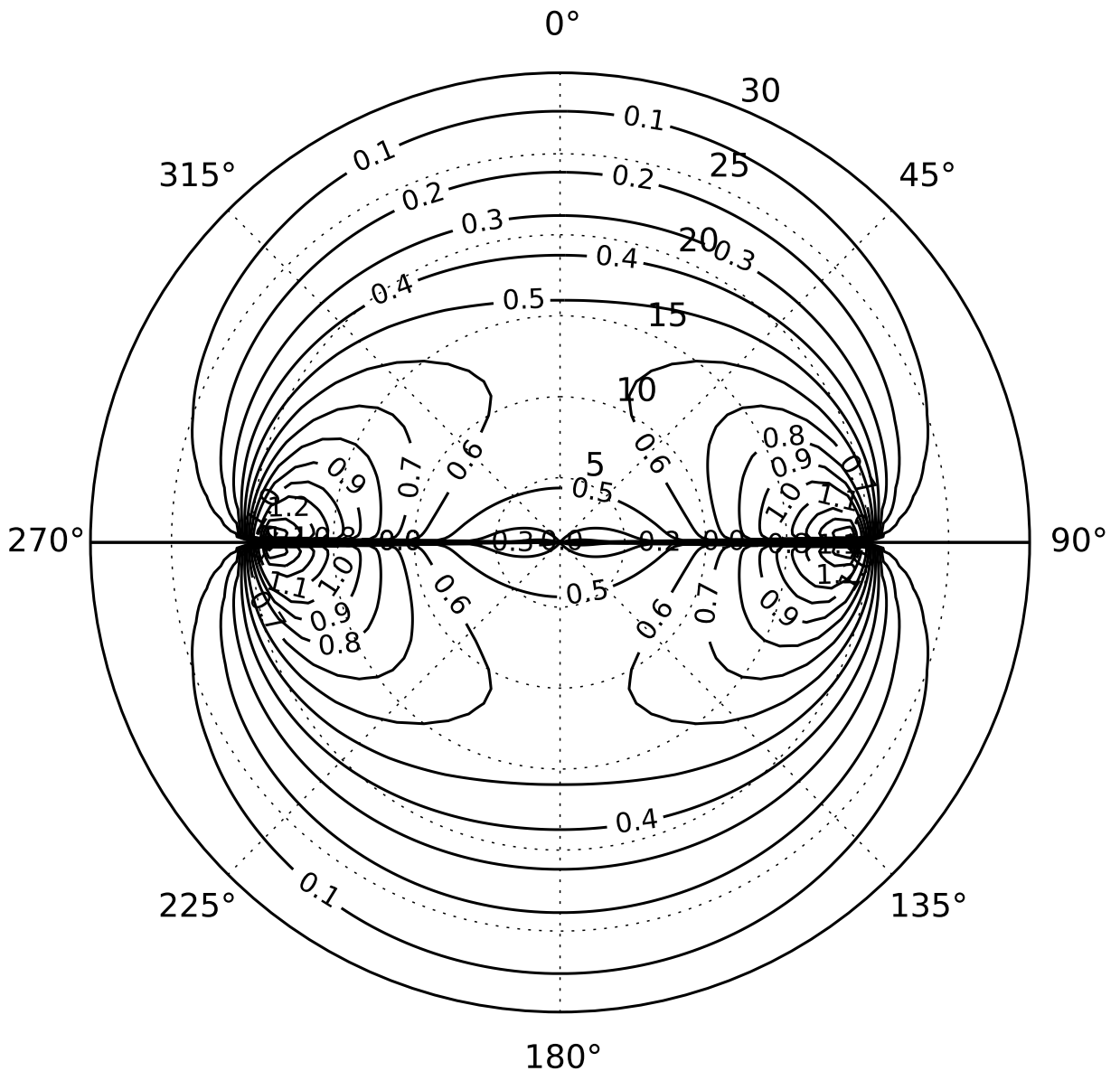

Fig. 3. Isolines of the azimuthal correction factor of the gravitational potential, $C_{\psi}$. The coordinates are the same as in Figure 1. Similarly to the gravitational potential, in the outer region free of isolines the value of $C_{\psi}$ asymptotically approaches to zero.

From (90) and (91) for the neutrino trajectories in the polar coordinates we obtain the following motion equations

$$
\ddot{D}-D \dot{\psi}^{2}=-\frac{\partial \Phi}{\partial D}
$$

and

$$
\frac{d(D \dot{\psi})}{d t}=D \ddot{\psi}+2 \dot{D} \dot{\psi}=-\frac{1}{D} \frac{\partial \Phi}{\partial \psi}
$$

In (92) there appears the centrifugal acceleration and in (93) the Coriolis acceleration term in the polar coordinates.

If the potential is expressed in the Cartesian coordinates then transition to the 


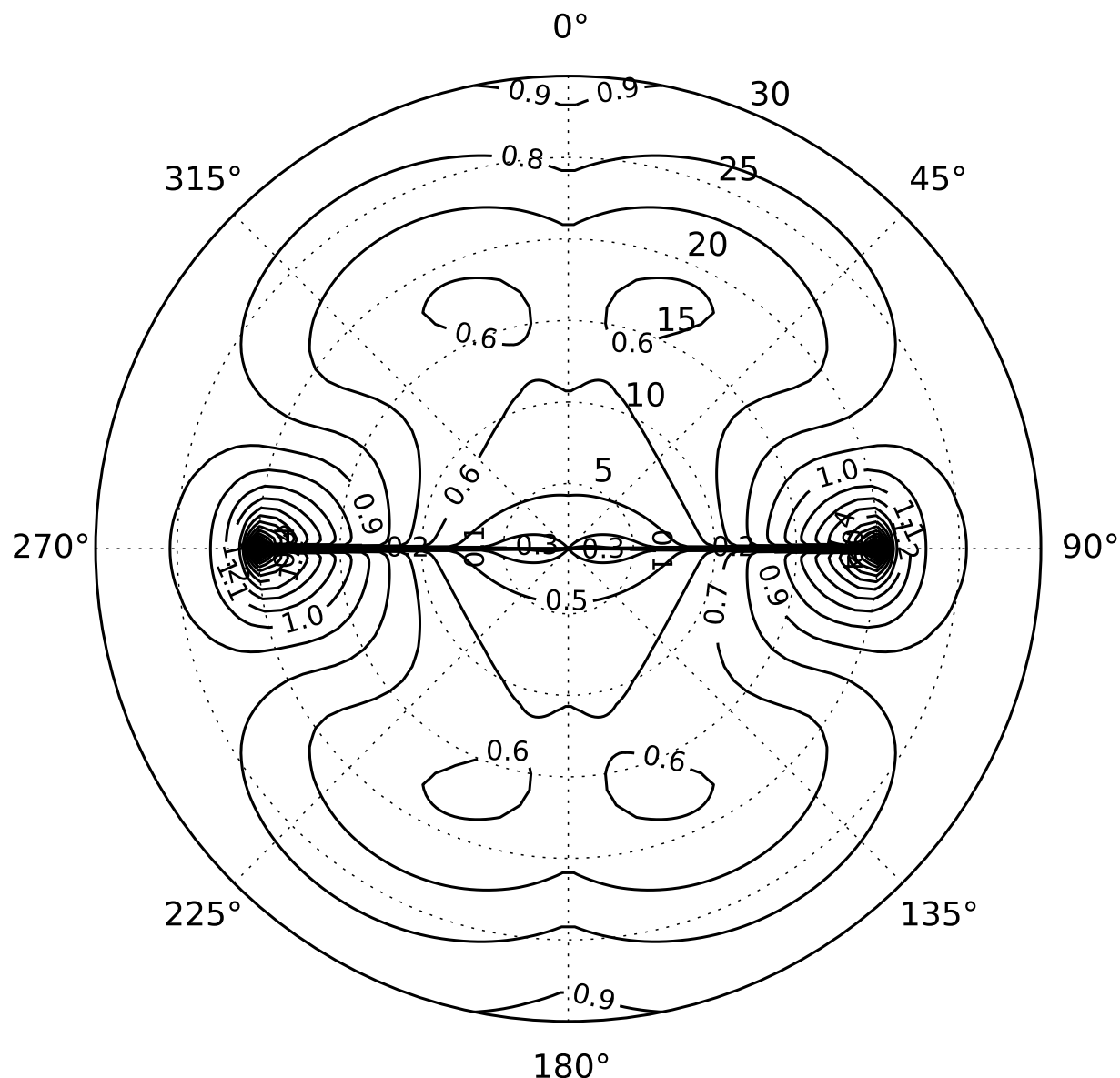

Fig. 4. Isolines of the correction factor of gravity, $C_{g}$. The coordinates are the same as in Figure 1. Similarly to the gravitational potential, in the outer region free of isolines the value of $C_{D}$ asymptotically approaches to 1.0 , and the central value of the correction factor is zero. The maximum correction factors are found on the galactic plane at $D=1$.

polar coordinates is accomplished by

$$
\frac{\partial \Phi}{\partial D}=\frac{\partial \Phi}{\partial x} \frac{\partial x}{\partial D}+\frac{\partial \Phi}{\partial z} \frac{\partial z}{\partial D}
$$

and

$$
\frac{\partial \Phi}{\partial \psi}=\frac{\partial \Phi}{\partial x} \frac{\partial x}{\partial \psi}+\frac{\partial \Phi}{\partial z} \frac{\partial z}{\partial \psi} .
$$

From the components of $\mathbf{D}$ we obtain

$$
\frac{\partial x}{\partial D}=\sin \psi, \quad \frac{\partial z}{\partial D}=\cos \psi
$$

and

$$
\frac{\partial x}{\partial \psi}=D \cos \psi, \quad \frac{\partial z}{\partial \psi}=-D \sin \psi
$$


Now we transform the differential equations of neutrino trajectories with time as argument to the form where arguments are the space coordinates and the functions are the velocities $\dot{D}$ and $\dot{\psi}$. Selection of the trajectory reduces the partial to total derivatives. The time derivative can be written as

$$
\frac{d}{d t}=\dot{D} \frac{\partial}{\partial D}+\dot{\psi} \frac{\partial}{\partial \psi}
$$

Replacing this operator in equations (92) and (93), for finding the velocity components we obtain the coupled partial differential equations

$$
\ddot{D}=\dot{D} \frac{\partial \dot{D}}{\partial D}+\dot{\psi} \frac{\partial \dot{D}}{\partial \psi}=-\frac{\partial \Phi}{\partial D}+D \dot{\psi}^{2}
$$

and

$$
\ddot{\psi}=\dot{D} \frac{d \dot{\psi}}{d D}+\dot{\psi} \frac{\partial \dot{\psi}}{\partial \psi}=-\frac{1}{D^{2}} \frac{\partial \Phi}{\partial \psi}-\frac{2 \dot{\psi} \dot{D}}{D}
$$

In addition the coupling of the equations for trajectories, being the characteristic curves of quasilinear partial differential equations, must hold

$$
\frac{d \psi}{d D}=\frac{\dot{\psi}}{\dot{D}}
$$

More generally for this case we obtain the system of Lagrange-Charpit equations in the form

$$
\frac{d D}{\dot{D}}=\frac{d \psi}{\dot{\psi}}=\frac{d \dot{D}}{\ddot{D}}=\frac{d \dot{\psi}}{\ddot{\psi}}=d \tau
$$

The expressions for $\ddot{D}$ and $\ddot{\psi}$ must be taken from (99) and (100), respectively. These three differential equations must be solved starting from the boundary values $\psi_{a}, \dot{D}_{a}, \dot{\psi}_{a}$ and $D_{a}$, all given on the accretion sphere surface.

In addition, we can use the energy conservation law

$$
\dot{D}^{2}+D^{2} \dot{\psi}^{2}=\dot{D}_{a}^{2}+D_{a}^{2} \dot{\psi}_{a}^{2}+2\left(\Phi_{a}-\Phi\right),
$$

being an integral of these equations. We can use it instead of (100).

Now for finding the density of neutrinos we must use the hydrodynamical mass conservation equation for neutrinos

$$
\frac{\partial\left(\rho v_{i}\right)}{\partial x_{i}}=0
$$

We must reduce this equation with partial derivatives relative of $\rho$ to full derivative expression along the neutrino trajectories.

In spherical coordinates, a general expression for the divergence of the neutrino flux can be written in the form

$$
\frac{1}{D^{2}} \frac{\partial\left(\rho D^{2} v_{D}\right)}{\partial D}+\frac{1}{D \sin \psi} \frac{\partial\left(\rho v_{\varphi}\right)}{\partial \varphi}+\frac{1}{D \sin \psi} \frac{\partial(\rho \sin \psi D \dot{\psi})}{\partial \psi}=0 .
$$


The velocity components here are

$$
v_{D}=\dot{D}, \quad v_{\psi}=D \dot{\psi}
$$

The equation of continuity (105) for the neutrinos, taking into account that in the case studied here it has no dependence on $\varphi$, takes the form

$$
\dot{D} \frac{\partial \rho}{D}+\dot{\psi} \frac{\partial \rho}{\psi}=\dot{\rho}
$$

where

$$
\dot{\rho}=-\frac{\rho}{D}\left(\frac{\partial\left(D^{2} \dot{D}\right)}{D \partial D}+\frac{D}{\sin \psi} \frac{\partial(\sin \psi \dot{\psi})}{\partial \psi}\right)
$$

Now to the system of the Lagrange-Charpit equations the following equation must be added:

$$
\frac{d \rho}{\dot{\rho}}=d \tau .
$$

The system of usual Lagrange-Charpit differential equations is to be solved by some traditional method.

In this section the change of gravities due to the neutrino background in circular disk galaxies has not been taken into account. This means that the equations are correct only in the case of relatively weak neutrino contribution. Nevertheless, the main features of the neutrino field remain probably the same if it is stronger. For the strong neutrino contribution, the only difference in the equations is that the potential due to neutrinos must be taken into account as additive in $\Phi$.

\section{BOUNDARY CONDITIONS FROM THE COSMIC NEUTRINO BACKGROUND}

The equations for neutrino trajectories and the density distribution are to be solved starting from the boundary conditions, integrating over the manifold of boundary data and in this way finding the total neutrino mass density distribution in the model galaxy studied.

The boundary values of space coordinates are specified trivially on the specified accretion sphere surface. The boundary velocities can be specified as velocity distribution on the accretion sphere by

$$
\dot{\mathbf{r}}_{a}=v_{a}\left(\sin \varphi_{a} \sin \psi_{a}, \cos \varphi_{a} \sin \psi_{a}, \cos \psi_{a}\right) .
$$

Using the same coordinate system as in the former sections, we obtain for the $z$-component of the angular momentum:

$$
L_{z}=x_{a} \dot{y}_{a}=x_{a}^{2} \dot{\varphi}_{a} .
$$

Integration must be carried out over boundary angles $\varphi_{a}$ and $\psi_{a}$ and over the velocities $v_{a}$. All of these values generate overlapping contributions to the total neutrino constituent in galaxies. Naturally, the boundary conditions chosen here are very tentative ones. The real situation is formed in the evolutionary processes of galaxies, which evidently favors also generation of the captured neutrino contribution, which in the present paper has not been studied. 
In flat circular axisymmetrical galaxies the density of neutrinos at a given distance from the galactic center increases from the equatorial surface to the polar axis due to lower velocities at given distance $D$ in the axial direction. This shows that the dark mass density in such galaxies must increase to the polar direction. The size of the central part of galaxies, where the velocities of neutrinos are essentially different from the radial ones, depends on the details of the neutrino flux densities.

In principle, study of stellar orbital velocities enables to obtain information about the neutrino velocities both in the cosmic background and locally in the galaxies studied. However, to make detailed conclusions about the neutrino distribution in galaxies, more additional studies are needed.

\section{SIMPLE AXISYMMETRICAL FLAT BARYON GALAXY MODELS}

To understand better the peculiarities of the possible cosmic neutrino background in galaxies, it is reasonable to propose simple approximations to typical axisymmetrical galaxies. Such models we name the toy model galaxies. The most simple model galaxy for study of neutrinos is a homogeneous circular disk. The next toy model we specify is an ellipsoidal model galaxy of constant density. This means that the mass of any disk $M^{\prime}$ in such a model is given by the equation

$$
M^{\prime}=\zeta \pi X^{2},
$$

where $\zeta$ is a constant, and the radii of the coaxial disks are specified by the equation of ellipse

$$
X^{2}=a^{2}-\frac{a^{2} Z^{2}}{b^{2}}
$$

Here the quantities $X$ and $Z$ are the gravity source coordinates in a baryonic galaxy, $a$ is the size of the larger and $b$ - of the smaller semi-axis of the ellipsoid. The ratio $b / a$ characterizes the ellipsoidality of the toy model galaxy studied. We have not studied dynamics of the baryonic component of galaxies. It is clear that our toy model galaxies in the present context are only an instrument for study of the main features of the neutrino contribution in galaxies.

\section{CONCLUDING REMARKS}

Since it is not fully excluded that the neutrinos can have the rest mass about 1 to $2 \mathrm{eV}$, they can be even a dominant constituent of the dark matter. However, the results obtained can be equally used for the analysis of other hypothetical weak-interacting and slow-moving non-relativistic particles in galaxies. We emphasize that a similar expansional cooling of non-relativistic baryons has occurred much earlier than for neutrinos, which enabled and accelerated considerably the formation of galaxies.

Due to the quadratic redshift in energy of massive non-relativistic cosmological neutrinos, the present neutrino background turns out to be almost completely degenerated and having extremely low temperature. The neutrinos have low velocities what favors to their important role in the formation and evolution of galaxies.

We studied the structure of the spherical galaxies, consisting of the baryonic matter supplemented by the cosmic neutrino background. The equations were derived for the gravitational potential and the gravity vector components of such galaxies, suggesting the presence in them of a wide equipotential region. 
For the circular disk galaxies of constant density we have derived relatively simple equations of their gravitational potential, its radial and azimuthal gravity components and also the total gravity. The ratios of these quantities relative to the point gravitation field are visualized in figures.

The equations of the neutrino trajectories in galaxies and of the mass density of the neutrino component from the mass conservation law have been derived. We also obtained the Lagrange-Charpit equations of this interconnected system of quasilinear partial differential equations.

Due to the circumstance that neutrino velocities are larger at larger potential values, a tendency appears to generate more spherical neutrino background and therewith wide potential wells in the axisymmetrical circular disk galaxies and the extended potential wells. This can be considered as a testimony that the dark mass can consist dominantly of cosmic background neutrinos.

In the present paper only the equations for computing of neutrino contribution to spherical and axisymmetrical circular disk galaxies have been derived, and their main features were tentatively discussed. The application of the derived equations to definite galaxies requires more efforts.

ACKNOWLEDGMENTS. This paper was partly supported by the research project SF0060030s08 of the Estonian Ministry of Education and Research. I am grateful to R. Poolamäe for his contribution in computing and shaping of the figures, and presenting the correction factors of the gravitational field.

\section{REFERENCES}

Canetti L., Drewes M., Shaposhnikov M. 2013, Physical Review Letters, 110, ID 061601

Conway J. T. 2000, MNRAS, 316, 540

Gupta A., Mathur S., Krongold Y., Nicastro F., Galeazzi M. 2012, ApJ, 756, L8

Huré J.-M., Hersant F. 2011, A\&A, 531, A36

Lass H., Blitzer L. 1983, Celestial Mechanics, 30, 225

Sapar A. 2011, Baltic Astronomy, 20, 267

Sapar A. 2013, Baltic Astronomy, 22, 315 